\title{
Clumps in stellar winds
}

\author{
J. S. Vink \\ Armagh Observatory, College Hill, BT61 9DG Armagh, Northern Ireland, UK \\ Correspondence to: J. S. Vink (jsv@arm.ac.uk)
}

Received: 29 April 2014 - Revised: 17 June 2014 - Accepted: 18 June 2014 - Published: 30 July 2014

\begin{abstract}
We discuss the origin and quantification of wind clumping and mass-loss rates $(\dot{M})$, particularly in close proximity to the Eddington $(\Gamma)$ limit, relevant for very massive stars (VMS). We present evidence that clumping may not be the result of the line-deshadowing instability (LDI), but that clumps are already present in the stellar photosphere.
\end{abstract}

\section{Introduction}

We address the issue of small-scale structures, or clumps, in radiatively-driven winds of massive stars. Stellar winds are important contributors to the kinetic energy input into the interstellar medium (ISM) - dominating supernovae for the first 10 Myrs (Voss et al., 2009). They are also relevant for the direct and indirect input of various chemical elements into the ISM. The indirect aspect involves the fact that $\dot{M}$ is a crucial parameter for the evolution of massive stars. For VMS in the range $60-300 M_{\odot}$ the winds become optically thick, showing Wolf-Rayet-type spectra of WNh-type, with both nitrogen $(\mathrm{N})$ and hydrogen $(\mathrm{H})$ present in emission. For VMS the effects of mass loss on evolution are even stronger. As VMS are thought to evolve close to chemically homogeneously (Gräfener et al., 2011) their ultimate fate is almost entirely determined by stellar wind mass loss (Yungelsen et al., 2008; Yusof et al., 2013; Köhler et al., 2014).

\section{Smooth radiation-driven wind models}

For optically thin O-star winds there are two distinct approaches in use to compute the opacity $\kappa$ and the resulting radiative acceleration $g_{\text {rad }}$. In the first one, the line acceleration is expressed as a function of the velocity gradient $(\mathrm{d} v / \mathrm{d} r)$ in the CAK theory of Castor et al. (1975). In the second approach, the line acceleration is expressed as a function of radius $r$ only (Müller and Vink, 2008; Muijres et al., 2012), which has been implemented in Monte Carlo models, which are ideally suited for studying clumping effects (Sects. 3 and 4) and the transition from optically thin to thick winds (Sect. 5) - relevant for VMS mass loss.

\section{Instabilities and wind clumping}

Because of the non-linear character of the equation of motion, the CAK solution is complex, with the $(\mathrm{d} v / \mathrm{d} r)$ physics involving instabilities due to the line-deshadowing instability LDI (see Owocki et al., 1988). One of the key implications of the LDI is that the time-averaged $\dot{M}$ is not anticipated to be affected by wind clumping, as it has the same average $\dot{M}$ as the smooth CAK solution. However, the shocked velocity structure and its associated density structure are expected to result in effects on the mass-loss diagnostics. In particular $\dot{M}$ diagnostics that is dependent on the square of the density, such as $\mathrm{H} \alpha$, will result in a square-root reduction of $\dot{M}$. However, ultraviolet P Cygni lines such as $\mathrm{Pv}$ are insensitive to clumping, as they depend linearly on density. Most analyses have been based on the assumption of optically thin clumps, but in reality, clumped winds are porous, with a range of clump sizes, masses, and optical depths. Oskinova et al. (2007) employed an effective opacity concept for line-profile modelling of the O-supergiant $\zeta$ Pup. Figure 1 shows that the most pronounced effect involves strong resonance lines, such as $\mathrm{Pv}$, which can be reproduced by their macro-clumping approach.

\section{Quantifying the number of clumps}

In the traditional view of line-driven winds of O-type stars via the CAK theory and the associated LDI, clumping would be expected to develop in the wind when the wind velocities are large enough to produce shocked structures. For typical O-star winds, this is thought to occur at about half the terminal wind velocity at about 1.5 stellar radii. Observational 

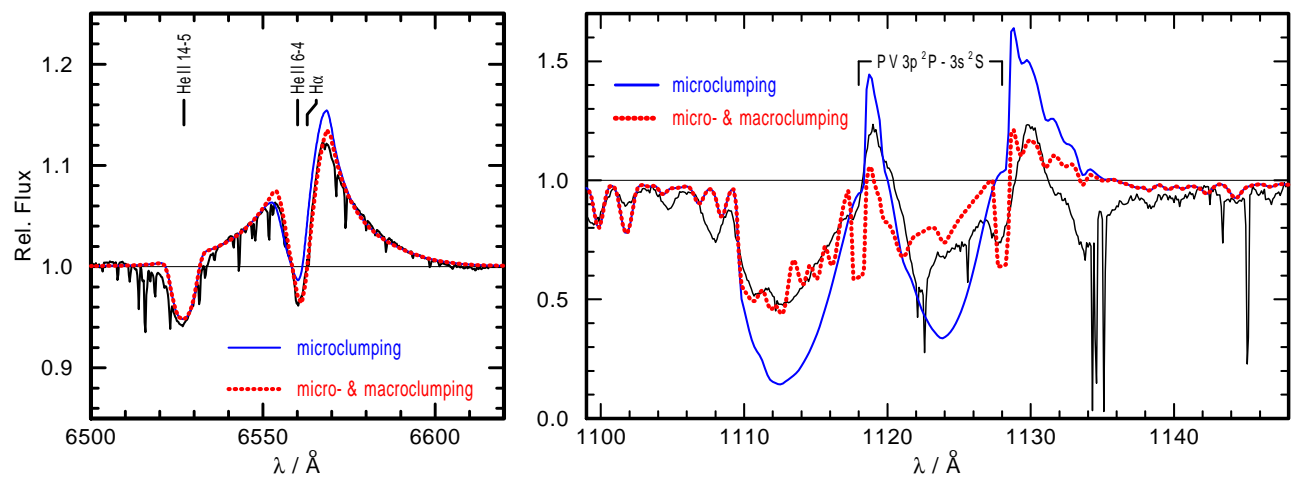

Figure 1. Ultraviolet Pv line and optical $\mathrm{H} \alpha$ line of the O-type supergiant $\zeta$ Pup. The black solid line indicates the observed spectrum. The blue solid line provides a P Cygni absorption that is much too strong. This is known as the Pv problem. However, when accounting for optically thick clumps and porosity (macro-clumping) the Pv line can be reproduced simultaneously with the H $\alpha$ line, without reducing $\dot{M}$. See the red dotted line. Source: Oskinova et al. (2007).

indications involving the existence of linear polarization (e.g. Davies et al., 2005) however show that clumping already shows up in the photosphere. Cantiello et al. (2009) suggested that waves produced by the sub-surface convection zone (associated with the OPAL Fe opacity peak) could cause wind clumping.

One may attempt to derive the number of convective cells by dividing the stellar surface area by the size of a convective cell. For main-sequence O-type stars, pressure scale heights are in a range $0.04-0.24 R_{\odot}$, corresponding to a total number of clumps of $6 \times 10^{3}-6 \times 10^{4}$ (see Davies et al., 2007; Cantiello et al,. 2009). These numbers may be tested through linear polarization variability observations. Davies et al. (2007) showed that more than half the luminous blue variables (LBVs) are intrinsically polarized. As the polarization angle was found to change irregularly with time, the observed line effects were considered to be the result of wind clumping.

An example of a model predicting the time-averaged polarization for the LBV P Cygni is shown in Fig. 2. There are two regimes where the observed polarization level may be reached. One is where the ejection rate is very low and only a few very optically thick clumps are ejected; the other scenario involves that of a very large number of clumps. The two scenarios may be distinguished via time-resolved polarimetry. Given the short timescale of the polarization variability data, we assume that LBV winds consist of thousands of clumps near the photosphere. However, for main-sequence O stars the derivation of wind-clump sizes from polarimetry has not yet been feasible as very high signal-to-noise data are required. LBVs offer an excellent opportunity for constraining the number of clumps from polarization variations due to the combination of higher mass-loss rates and lower terminal velocities. Davies et al. (2007) found that in order to produce the polarization variability of P Cygni, the wind should consist of some 1000 clumps per wind flow-time. In order to check whether this is compatible with subsurface convec-

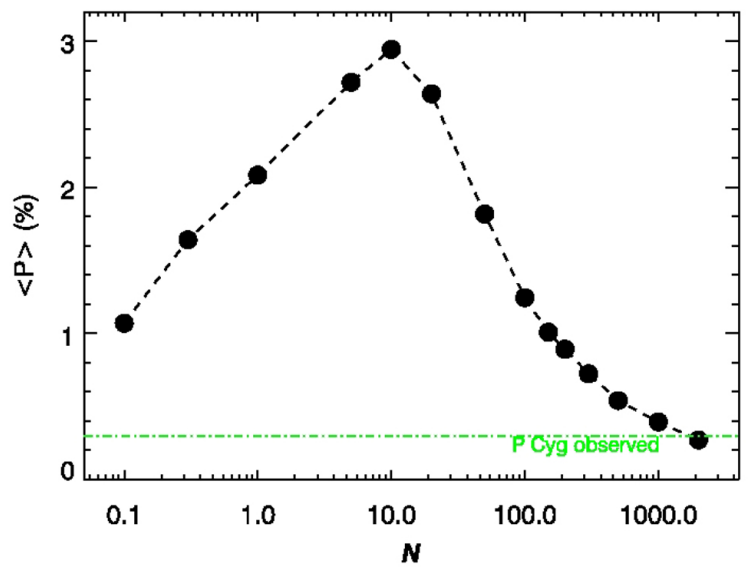

Figure 2. Time-averaged polarization over a range of ejection rates (per wind flow-time). At $\mathcal{N} \sim 20$, the optical depth per clump exceeds unity and the overall polarization drops (see Davies et al., 2007 for details). The observed amount of polarization is indicated by the dash-dotted line. Note that there are two ejection-rate regimes (one at high $\mathcal{N}$ and one at low $\mathcal{N}$; the latter based on extrapolation) where the observed level of polarization may be achieved for P Cygni.

tion being the root cause for clumping, we would need to consider the sub-surface convective regions of an object with properties similar to those of P Cygni. Because of the lower gravity of PCyg the pressure scale height is about $4 R_{\odot}$, i.e. significantly larger than for O-type stars. Therefore, the same estimate for the number of clumps as done for the $\mathrm{O}$ stars yields some 500 clumps per wind-flow time, which is consistent with that derived from the polarization data!

\section{Enhanced mass loss for VMS}

In Fig. 3 we show mass-loss predictions for VMS as a function of Eddington parameter $\Gamma$, where $\Gamma=\frac{\kappa L}{4 \pi c M}$ basically represents the $L / M$ ratio. Vink et al. (2011) showed that 


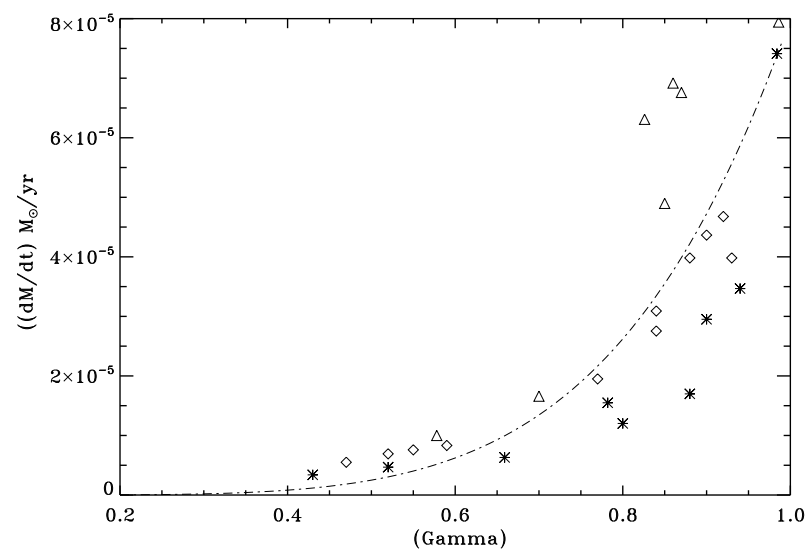

Figure 3. Mass-loss predictions versus the Eddington parameter $\Gamma$, with $\Gamma=\frac{\kappa L}{4 \pi c M}$ representing the $L / M$ ratio. The various symbols correspond to models within different mass ranges, which can be found in Vink et al. (2011). The dashed dotted line indicates a very steep relation between $\dot{M}$ and $\Gamma^{x}$ with $x=5$.

for normal O-type stars that have a relatively low $\Gamma$ and thin winds, the $\dot{M} \propto \Gamma^{x}$ relationship is rather shallow, with $x \simeq 2$. However, they also show there is a steepening at high $\Gamma$, where $x$ becomes as large as $\simeq 5$. Here the objects show optically thick WNh-like winds, with wind optical depth $(\tau)$ and wind efficiency $(\eta)$ crossing unity. Note that the wind efficiency $\eta=\frac{\dot{M} v_{\infty}}{L / c}$ provides the ratio between the momentum gained by the gas over that from the radiation field (per second). Gräfener et al. (2011) and Bestenlehner et al. (2014) recently provided empirical evidence for a steep exponent $(x \simeq 5)$. We have also calibrated the Vink et al. (2000) rates using an analytic method - involving $\eta=\tau=1$ - and applied it to the most massive stars in the Arches cluster (Vink and Gräfener, 2012).

Using the canonical Vink et al. (2000) rates for a star with some $300 M_{\odot}$ we obtain $\dot{M}=10^{-4.2} M_{\odot}$ year $^{-1}$. For a lifetime of some 2.5 million years (Yungelsen et al., 2008; Yusof et al., 2013; Köhler et al., 2014) this would result in a total main-sequence mass lost of $150 M_{\odot}$. Additional mass loss during helium burning is expected to further "evaporate" the object. In other words there is little room for substantial amounts of mass loss through alternative modes, such as eruptive mass loss or Roche-lobe overflow. Finally, our results imply that pair-instability supernovae are unlikely to exist locally, unless we consider low $Z$ galaxies.

Edited by: H. Fichtner

Reviewed by: two anonymous referees

\section{References}

Cantiello, M., Langer, N., Brott, I., de Koter, A., Shore, S. N., Vink, J. S., Voegler, A., Lennon, D. J., and Yoon, S.-C.: Sub-surface convection zones in hot massive stars and their observable consequences, Astron. Astrophys., 499, 279-290, 2009

Castor, J. I., Abbott, D. C., and Klein, R. I.: Radiation-driven winds in Of stars, Astrophys. J., 195, 157-174, 1975.

Davies, B., Oudmaijer, R. D., and Vink, J. S.: Asphericity and clumpiness in the winds of Luminous Blue Variables, Astron. Astrophys., 439, 1107-1125, 2005.

Davies, B., Vink, J. S., Oudmaijer, R. D.: Modelling the clumpinginduced polarimetric variability of hot star winds, Astron. Astrophys., 469, 1045-1056, 2007.

Gräfener, G., Vink, J. S., de Koter, A., and Langer, N.: The Eddington factor as the key to understand the winds of the most massive stars - Evidence for a $\Gamma$-dependence of Wolf-Rayet type mass loss, Astron. Astrophys., 535, A56, doi:10.1051/00046361/201116701, 2011.

Muijres, L. E., Vink, J. S., de Koter, A., Müller, P. E., and Langer, N.: Predictions for mass-loss rates and terminal wind velocities of massive O-type stars, Astron. Astrophys., 537, A37, doi:10.1051/0004-6361/201015818, 2012.

Müller, P. E. and Vink, J. S.: A consistent solution for the velocity field and mass-loss rate of massive stars, Astron. Astrophys. 492, 493-509, 2008.

Oskinova, L. M., Hamann, W.-R., and Feldmeier, A.: Neglecting the porosity of hot-star winds can lead to underestimating mass-loss rates, Astron. Astrophys., 476, 1331-1340, 2007.

Owocki, S. P., Castor, J. I., and Rybicki, G. B.: Time-dependent models of radiatively driven stellar winds. I - Nonlinear evolution of instabilities for a pure absorption model, Astrophys. J., 335, 914-930, 1988.

Vink, J. S. and Gräfener, G.: The transition mass-loss rate: Calibrating the role of line-driven winds in massive star evolution, Astrophys. J., 751, L34, doi:10.1088/2041-8205/751/2/L34, 2012.

Vink, J. S., de Koter, A., Lamers, H. J. G. L. M.: New theoretical mass-loss rates of O and B Stars, Astron. Astrophys., 362, 295309, 2000.

Vink, J. S., Muijres, L.E., Anthonisse, B., de Koter, A., Gräfener, G., and Langer, N.: Wind modelling of very massive stars up to 300 solar masses, Astron. Astrophys., 531, A132, doi:10.1051/00046361/201116614, 2011.

Voss, R., Diehl, R., Hartmann, D. H., Cerviño, M., Vink, J. S., Meynet, G., Limongi, M., and Chieffi, A.: Using population synthesis of massive stars to study the interstellar medium near OB associations, Astron. Astrophys., 504, 531-542, 2009.

Yungelson, L. R., van den Heuvel, E. P. J., Vink, J. S., Portegies Zwart, S. F., and de Koter, A.: On the evolution and fate of supermassive stars, Astron. Astrophys., 477, 223-237, 2008.

Yusof, N., Hirschi, R., Meynet, G., Crowther, P. A., Ekström, S., Frischknecht, U., Georgy, C., Abu Kassim, H., and Schnurr, O.: Evolution and fate of very massive stars, Mon. Not. Roy. Astron. Soc., 433, 1114-1132, 2013. 